\title{
Spindle cell oncocytoma of the sella turcica with anaplastic features and rapid progression in short-term follow-up: a case report with proposal of distinctive radiologic features
}

\author{
Dong Ja Kim ${ }^{1}$, SangHan Lee ${ }^{2}$, Mee-seon $\mathrm{Kim}^{3}$, Jeong-Hyun Hwang ${ }^{4}$, Myong Hun Hahm ${ }^{5}$ \\ 'Department of Forensic Medicine, School of Medicine, Kyungpook National University, Daegu; \\ ${ }^{2}$ Department of Forensic Medicine, Defense Institute of Forensic Science, Seoul; \\ ${ }^{3}$ Department of Pathology, Kyungpook National University Hospital, Daegu; \\ Departments of ${ }^{4}$ Neurosurgery and ${ }^{5}$ Radiology, School of Medicine, Kyungpook National University, Daegu, Korea
}

\begin{abstract}
We present a rare case of spindle cell oncocytoma (SCO) of the sella turcica with malignant histologic features and rapid progression. A 42-year-old woman experienced bilateral blurred vision and was preoperatively misdiagnosed as having a pituitary macroadenoma on magnetic resonance imaging. After surgery, SCO was diagnosed by the histopathologic features of interlacing fascicles of spindle tumor cells with finely granular, eosinophilic cytoplasm. Focal anaplastic changes and necrosis were present. Immunohistochemically, the tumor cells were positive for vimentin, epithelial membrane antigen, S-100, galectin-3, and thyroid transcription factor 1 . Four months later, the tumor had progressed, and second surgery with adjuvant radiotherapy was performed; the patients remains under observation. In this report, we proposed distinctive radiologic features for differential diagnosis between SCO and other pituitary tumors.
\end{abstract}

Key Words: Oncocytoma; Sella turcica; Neoplasms; Progression; Radiology

Received: December 1, 2020 Revised: January 14, 2021 Accepted: January 26, 2021

Corresponding Author: Myong Hun Hahm, Department of Radiology, School of Medicine, Kyungpook National University, 680 Gukchaebosang-ro, Jung-gu, Daegu 41944, Korea

Tel: +82-53-200-3376/3380, Fax: +82-53-200-3349/3969, E-mail: hammh7@gmail.com

In 2002, Roncaroli et al. [1] initially described spindle cell oncocytoma (SCO) of the sella turcica, which has been characterized as World Health Organization (WHO) grade I non-endocrine neoplasm of the sella turcica [2]. From 2002 to 2019, <50 cases of SCO of the sella turcica had been reported in the literature as case reports or case series $[1,3,4]$. Histologically, this tumor is composed of fascicles of spindle cells with eosinophilic oncocytic cytoplasm. The tumor cells lack immunoreactivity for neuroendocrine markers and pituitary hormones. Mitoses are rare in most cases, and SCO was initially described with a benign clinical course [1]. However, subsequent cases with multiple recurrences have been reported; therefore, differential radiologic diagnosis from other benign pituitary neoplasm is important.

Herein, we present a preoperatively misdiagnosed case of SCO of the sella turcica with focal anaplastic histologic features and rapid progression in the short-term follow-up period, suggesting a malignant clinical course. Moreover, we retrospectively proposed a distinctive radiologic finding of $\mathrm{SCO}$ on preoperative magnetic resonance imaging (MRI).

\section{CASE REPORT}

A 42-year-old woman presented with complaints of bilateral blurred vision for 3 months. Bitemporal hemianopsia was observed on ophthalmological examination. A 22-mm-sized sella/ suprasellar mass was found on brain MRI. The mass had wellcircumscribed margin, compression at the optic chiasm, and intense enhancement without gross hemorrhage, calcification, and necrosis. As will be described later in the Discussion, proposed characteristic findings of SCO of the sella turcica, which were different from those of pituitary adenoma, were observed in dynamic contrast enhanced T1-weighted imaging (DCE-T1WI) as follows: (1) spoke-wheel pattern of early arterial enhancement in periphery and (2) late central stellate enhancement (Fig. 1). 
On preoperative evaluation, there was no abnormality in other organs. The patient had been taking medication for hypothyroidism and adrenal insufficiency before visiting our hospital. Preoperative laboratory tests in our hospital revealed normal range of thyroid, cortisol, and adrenocorticotropic hormone; T3, 0.74 ng/mL (0.6-1.9); free T4, $1.47 \mathrm{ng} / \mathrm{dL}$ (0.89-1.8); cortisol, 9.19 ng/dL (2.47-19.5); and adrenocorticotropic hormone, $29.44 \mathrm{pg} /$ $\mathrm{mL}(0-60)$. Abnormal results of hormonal test included low thyroid-stimulating hormone level at $0.07 \mu \mathrm{IU} / \mathrm{mL}(0.3-0.4)$, elevated prolactin level at $48.85 \mathrm{ng} / \mathrm{mL}(4.79-23.3)$, and low luteinizing hormone level at $0.38 \mathrm{mIU} / \mathrm{mL}(0.56-89.09)$.

She was initially misdiagnosed as having pituitary adenoma or craniopharyngioma and underwent endoscopic transnasal trans- sphenoidal resection. The neurosurgeon described the tumor as highly vascular and causing significant intraoperative bleeding. Therefore, total resection was not possible, and residual solid lesion was observed in the superior part of the mass on immediate postoperative MRI (Fig. 2).

Grossly, the tumor mass was pale-yellow and solid with focal hemorrhage. Histologically, the tumor was mainly composed of interlacing fascicles of plump spindle cells with eosinophilic and oncocytic cytoplasm and intervening thin blood vessels. Mild to moderate nuclear atypia was identified, and focal pleomorphic tumor cells were noted. Mitoses were frequent (3-4 counts/10 high-power field), and focal coagulative necrosis was seen (Fig. $3 \mathrm{~A}-\mathrm{C})$. The immunohistochemical study revealed a lack of re-
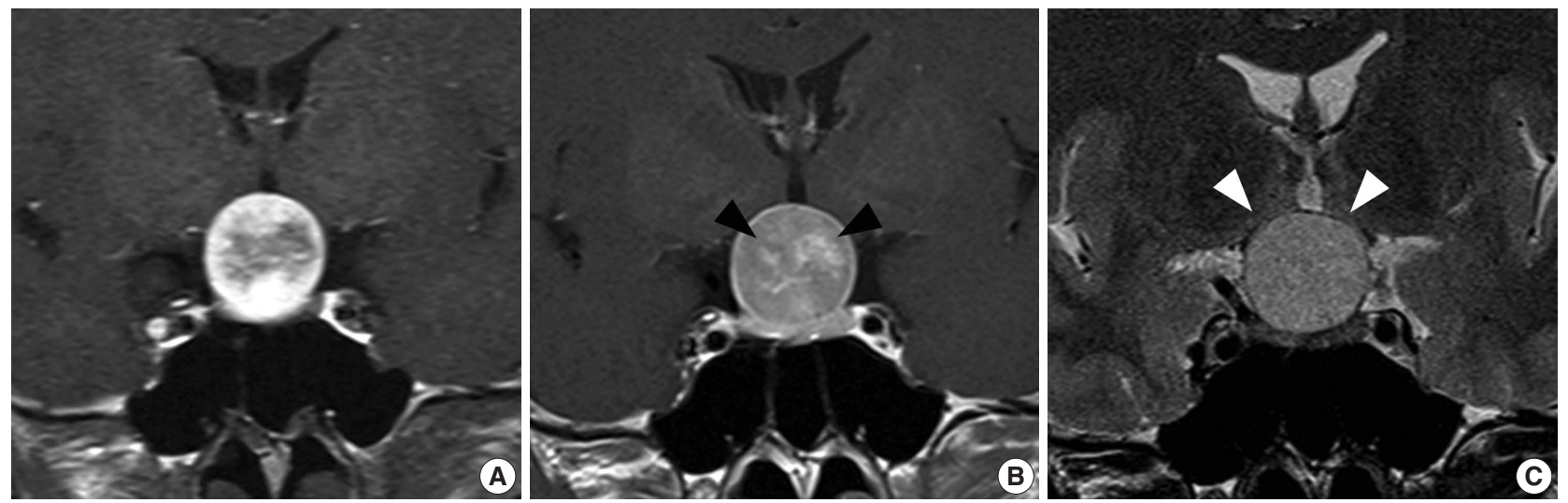

Fig. 1. Preoperative magnetic resonance imaging. Coronal dynamic contrast enhanced T1-weighted imaging, early arterial phase (A), delayed phase (B), and coronal T2-weighted imaging (C). Note the late central stellate enhancement (black arrowheads). White arrowheads indicate the paper-thin compressed optic chiasm.
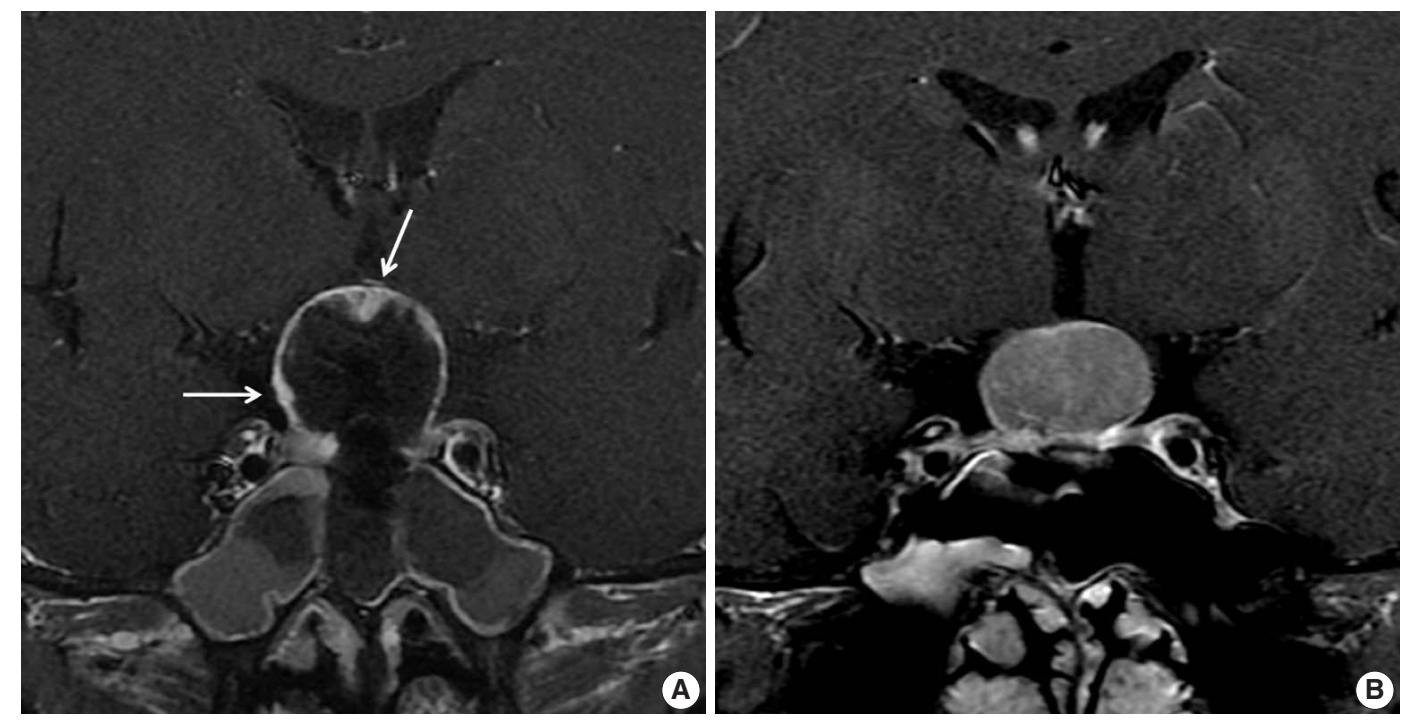

Fig. 2. Immediate postoperative contrast enhanced magnetic resonance imaging (MRI) (A) and 4-month follow-up MRI (B). Note the residual solid lesion including the superior part of the mass on immediate postoperative MRI (arrows) and rapid progression of the mass refilling the operation cavity. 

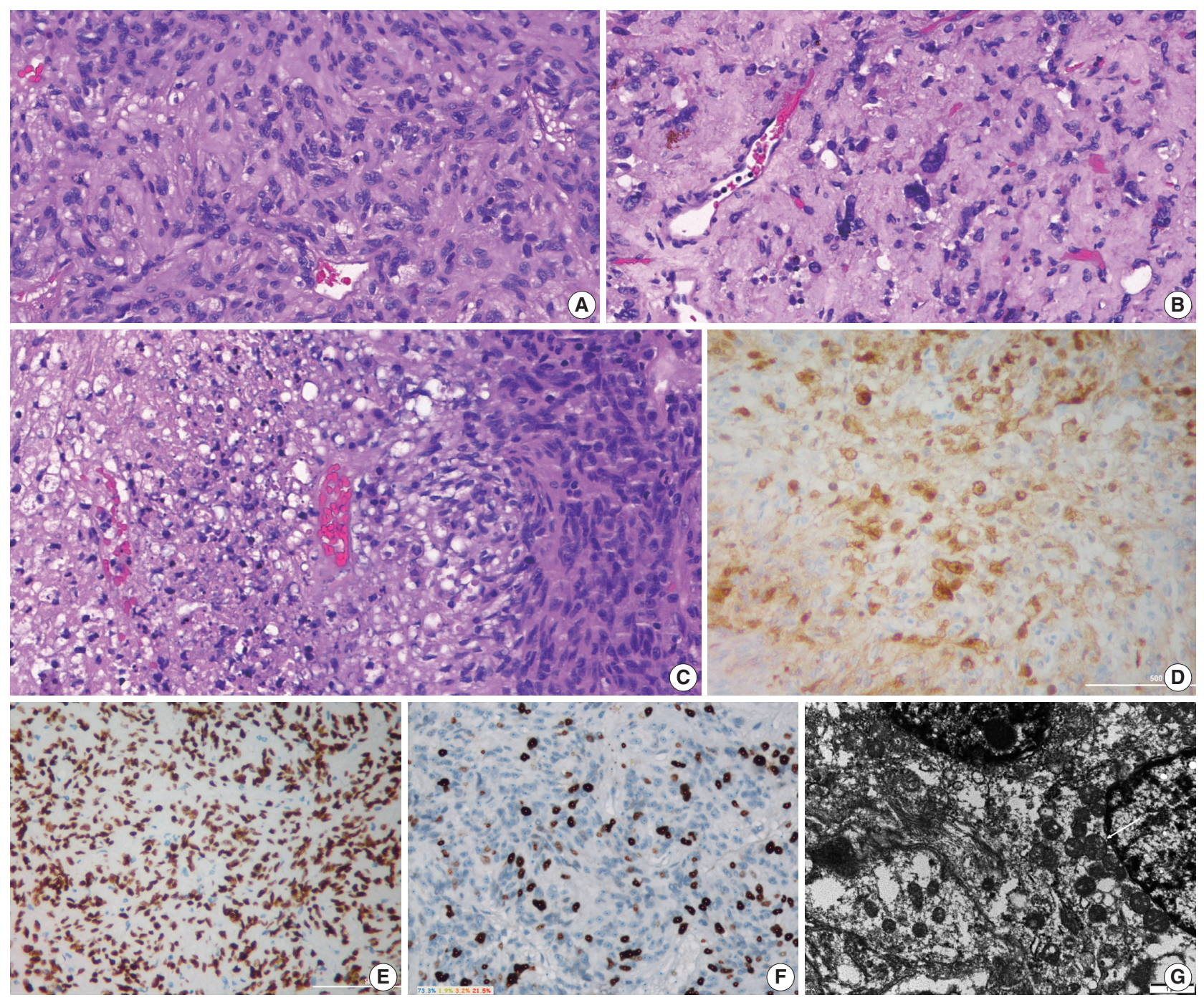

Fig. 3. Histopathologic findings. (A) The tumor is composed of interlacing fascicles of spindle cells with abundant eosinophilic cytoplasm. (B) Focal nuclear pleomorphism and hyalinized stroma are seen. (C) Focal coagulative tumor necrosis is present. The tumor cells show immunoreactivity for galectin-3 (D) and thyroid transcription factor 1 (E). (F) The Ki-67 index was 26.7\%. (G) Ultrastructural examination using paraffinembedded tissue block show numerous mitochondria (white arrow) in tumor cell.

activity for neuroendocrine markers and pituitary hormones. The tumor cells were immunoreactive for vimentin, epithelial membrane antigen (EMA), S-100 protein, thyroid transcription factor 1 (TTF-1), and galectin-3 (Fig. 3D, E) and negative for glial fibrillary acidic protein. The Ki-67 index was $26.7 \%$ (Fig. 3F). The tumor was diagnosed as SCO based on the characteristics of histopathology and immunohistochemical results. On ultrastructural findings using paraffin-embedded tissues, the neoplastic cells contained numerous mitochondria with lamellar cristae. The neoplastic cells were linked by intermediate junctions and desmosomes (Fig. 3G).

Four months after initial operation, the patient complained of headache and aggravating blurred vision of the left eye. Fol- low-up MRI revealed a regrown tumor mass (Fig. 2B), and the patient underwent a second surgery. Histologic sections of the materials from the second operation revealed similar pathologic features, and the Ki-67 index was 20.5\%. Postoperative adjuvant radiotherapy has been performed, and the patient is under observation.

\section{DISCUSSION}

SCO is a rare nonfunctioning tumor of the sella turcica and accounts for approximately $0.1 \%$ to $0.4 \%$ of all sellar regions tumors $[1,5,6]$. Previously, this tumor was suspected to be derived from folliculostellate cells of the anterior pituitary gland, which 
are sustentacular cells of adenohypophysis [1]. However, subsequent studies reported that pituicytes are suggested as their cells of origin. Owing to the same or similar immunohistochemical characteristics, Mete et al. [7] believed that SCO is a variant of pituicytoma. The term "pituicytoma" has been used to describe a number of tumors in the region of the sella turcica, such as pilocytic astrocytomas, granular cell tumors, and even pituitary adenoma. Nontumorous pituicytes, SCOs, and granular cell tumors are positive for TTF-1, but folliculostellate cells and adenohypophysis are negative for TTF-1, which indicates that SCO is a common lineage of pituicytes [7].

Clinically, the tumor is often misdiagnosed as a nonfunctioning pituitary adenoma. Because of the compressive effect on the optic chiasm and pituitary gland, the common symptoms include visual disturbances, headache, and panhypopituitarism. Histologically, the tumor is composed of spindle cells arranged in interlacing fascicular structures with intervening blood vessels and should be differentiated with null cell adenoma with oncocytic change, meningioma, schwannoma, granular cell tumor, solitary fibrous tumor, and paraganglioma [8]. Immunohistochemically, SCO is negative for neuroendocrine markers such as synaptophysin and chromogranin, pituitary hormones, cytokeratin, desmin, and smooth muscle actin and typically positive for TTF-1, EMA, vimentin, and galectin-3 [1,3-6]. The ultrastructural characteristics of SCO include cytoplasmic accumulation of numerous mitochondria and several cell-cell junctions, mainly short desmosomes [9].

Many authors have described the radiologic features of SCO of the sella turcica as nonspecific and generally not differentiable from pituitary adenoma. However, Hasiloglu et al. recently described new radiologic signs in diagnosis of SCO of the sella turcica in three cases: (1) millimetric hypointense foci and linear signal void areas in T2-weighted imaging and (2) hypervascular features of the tumor such as early intense contrast enhancement in DCE-T1WI [10]. Reported findings of SCO of the sella turcica are distinguishable from those of a typical pituitary adenoma characterized by later enhancement than a normal pituitary gland. However, these are less specific findings for SCO.

Interestingly, our case showed a common enhancement pattern of so-called segmental enhancement inversion. This is a spokewheel pattern of early arterial enhancement in the periphery and late central stellate enhancement and is typically observed in renal oncocytoma with a critical size, as summarized by Woo et al. [11]. Early arterial spoke-wheel-like enhancement is observed by peripheral parenchymal tissues composed of compactly arranged tumor cells with scarcely intervening stroma, and delayed central enhancement is attributed to a central fibrous stellate scar $[11,12]$. This radiopathologic evidence of a central fibrous stellate scar were reported in adrenal oncocytoma and thyroid oncocytic (Hurthle cell) tumor $[13,14]$.

In our case, radiopathologic correlation was not possible because of piecemeal resection owing to a surgical procedure, but peculiar radiologic findings were identified. Therefore, we propose that the central fibrous stellate scar causing segmental enhancement inversion is shared by oncocytoma and might be a highly specific finding for SCO of the sella turcica. Conclusively, we suggest that SCO could be diagnosed preoperatively.

Discrimination can be difficult even if distinctive MRI findings are recognized because SCO is a very rare tumor in the sella turcica. However, early arterial enhancement and signal void are predictors of high bleeding risk during surgery, regardless of histopathologic diagnosis. Therefore, considering the possibility of tumor other than benign adenoma, we should have discussed the option of preoperative embolization and should have attempted complete resection.

Fewer than 50 cases had been reported in the English literature. Giantini Larsen et al. [3] retrospectively reviewed 40 cases of SCO of the pituitary glands from 2002 to 2018 and additionally presented six cases in 2018. In 2019, Yip et al. [4] reported a case of SCO presenting as pituitary apoplexy. The ages of patients varied from 24 to 88 years. Because the preoperative diagnosis was suggestive of pituitary adenoma, the patients were often managed by transsphenoidal approach. When Roncaroli et al. [1] described five cases of SCOs in 2002, no recurrence was noted in any case within 2-68 months of follow-up. They suggested a benign nature of SCO based on the absence of cellular anaplasia, mitoses, and necrosis along with a low Ki-67 proliferation labeling index. In contrast to the initially described benign behavior, many authors have reported several cases of recurrent SCO. In their retrospective review, Giantini Larsen et al. [3] reported that $39 \%$ were recurrent at the time of surgery and recurred postoperatively after resection. However, SCO is retained as a grade I tumor in the recent updated 2017 WHO classification [2]. We believe that it is important to reevaluate the clinical outcome of this tumor in a large-scale study and to identify the histopathologic factors to predict aggressive behavior of this tumor. The Ki-67 proliferation index was low in most cases, with a range of $1 \%$ to $8 \%$ [3]. Kong et al. [15] reported a case of malignant SCO with repeating recurrence and a continuously increasing $\mathrm{Ki}-67$ index up to $45 \%$. In the present case, increased mitotic figures, nuclear atypia, necrosis, and high MIB-1 index are possible contributory factors for patient prognosis. 
The tumor progressed within 4 months after the operation, and the time interval from initial operation and tumor progression was very short. Thus, SCO is more clinically aggressive and should be classified as malignancy compared with other tumors of the sellar region. Although SCO of the sella turcica can have malignant behavior, no radiologic malignant features of SCO such as gross hemorrhage, necrosis, or invasion into the adjacent tissue was identified in our case. Hence, radiologically, SCO should be considered preoperatively with segmental enhancement inversion, and postoperative close follow-up is necessary.

The pathogenesis and prognosis of SCOs remain uncertain, and the behavior of this tumor needs to be further studied. Classification of SCO of the pituitary gland will establish more biologically and clinically uniform groups of tumors. This will allow pathologists and radiologists to better diagnose these tumors and predict clinical outcomes for the patients.

\section{Ethics Statement}

Formal written informed consent was not required with a waiver by the appropriate IRB//Ethics Committee (KNUH IRB No. 2020-09-029).

\section{Availability of Data and Material}

The datasets generated or analyzed during the study are available from the corresponding author on reasonable request.

\section{Code Availability}

Not applicable.

\section{ORCID}

Dong Ja Kim

SangHan Lee

Mee-seon Kim

Jeong-Hyun Hwang

Myong Hun Hahm

\begin{abstract}
https://orcid.org/0000-0001-8462-3173 https://orcid.org/0000-0003-0390-3494 https://orcid.org/0000-0002-4244-1985 https://orcid.org/0000-0002-5306-6922 https://orcid.org/0000-0001-9165-6117
\end{abstract}

\section{Author Contributions}

Conceptualization: Kim DJ, Lee S, Data curation: Kim DJ, Hahm MH. Formal analysis: Kim DJ, Lee S. Investigation: Kim MS. Methodology: Kim DJ, Hahm MH. Software: Kim DJ. Validation: Hahm MH. Visualization: Kim MS. Writing—original draft: Kim DJ, Hahm MH. Writing_review \& editing: Kim DJ, Hwang JH, Hahm MH.

\section{Conflicts of Interest}

The authors declare that they have no potential conflicts of interest.

\section{Funding Statement}

No funding to declare.

\section{Acknowledgments}

The authors thank Professor Sung-Hye Park, Department of Pathology, Seoul National University College of Medicine, for her diagnostic assistance with this study.

\section{References}

1. Roncaroli F, Scheithauer BW, Cenacchi G, et al. 'Spindle cell oncocytoma' of the adenohypophysis: a tumor of folliculostellate cells? Am J Surg Pathol 2002; 26: 1048-55.

2. Lopes MB. The 2017 World Health Organization classification of tumors of the pituitary gland: a summary. Acta Neuropathol 2017; 134: 521-35.

3. Giantini Larsen AM, Cote DJ, Zaidi HA, et al. Spindle cell oncocytoma of the pituitary gland. J Neurosurg 2018; 131: 517-25.

4. Yip CM, Lee HP, Hsieh PP. Pituitary spindle cell oncocytoma presented as pituitary apoplexy. J Surg Case Rep 2019; 2019: rjz179.

5. Kloub O, Perry A, Tu PH, Lipper M, Lopes MB. Spindle cell oncocytoma of the adenohypophysis: report of two recurrent cases. Am J Surg Pathol 2005; 29: 247-53.

6. Matyja E, Maksymowicz M, Grajkowska W, Olszewski W, Zielinski G, Bonicki W. Spindle cell oncocytoma of the adenohypophysis: a clinicopathological and ultrastructural study of two cases. Folia Neuropathol 2010; 48: 175-84.

7. Mete O, Lopes MB, Asa SL. Spindle cell oncocytomas and granular cell tumors of the pituitary are variants of pituicytoma. Am J Surg Pathol 2013; 37: 1694-9.

8. Sali A, Epari S, Tampi C, Goel A. Spindle cell oncocytoma of adenohypophysis: Review of literature and report of another recurrent case. Neuropathology 2017; 37: 535-43.

9. Guadagno E, Cervasio M, Di Somma A, Califano M, Solari D, Del Basso De Caro M. Essential role of ultrastructural examination for spindle cell oncocytoma: case report of a rare neoplasm and review of the literature. Ultrastruct Pathol 2016; 40: 121-4.

10. Hasiloglu ZI, Ure E, Comunoglu N, et al. New radiological clues in the diagnosis of spindle cell oncocytoma of the adenohypophysis. Clin Radiol 2016; 71: 937.

11. Woo S, Cho JY, Kim SH, et al. Segmental enhancement inversion of small renal oncocytoma: differences in prevalence according to tumor size. AJR Am J Roentgenol 2013; 200: 1054-9.

12. Monk IP, Lahiri R, Sivaprakasam R, Malhotra S, Praseedom RK, Jah A. Adrenocortical oncocytoma: review of imaging and histopathological implications. Int J Surg Case Rep 2010; 1: 30-2.

13. Coppola M, Romeo V, Verde F, et al. Integrated imaging of adrenal oncocytoma: a case of diagnostic challenge. Quant Imaging Med Surg 2019; 9: 1896-901.

14. Deng D, Chen X, Wang H, Wu H. Typical manifestations of Hurthle cell adenoma of the thyroid on contrast-enhanced CT: a case report. Medicine (Baltimore) 2019; 98: e15866.

15. Kong X, Li D, Kong Y, Zhong D. Malignant adenohypophysis spindle cell oncocytoma with repeating recurrences and a high Ki-67 index. Medicine (Baltimore) 2017; 96: e5657. 